\title{
Force Scanning on a Shaky Membrane
}

\author{
A microscope technique that visualizes small objects on a vibrating \\ membrane could deliver atomically resolved MRI scans.
}

\author{
By Michael Schirber
}

A new membrane-based force microscope provides a potential platform for an MRI scanner that could image a single virus. Researchers placed nanoscale objects on a vibrating membrane and moved a probe back and forth over the surface. They created images of the objects by monitoring the vibrations of the membrane, which revealed the force between the probe and the object at each location. The device can't yet beat the sensitivity of state-of-the-art systems, but with continued development, it could eventually measure the magnetic force from a single nucleus, which could lead to atomically resolved MRI images, according to the developers.

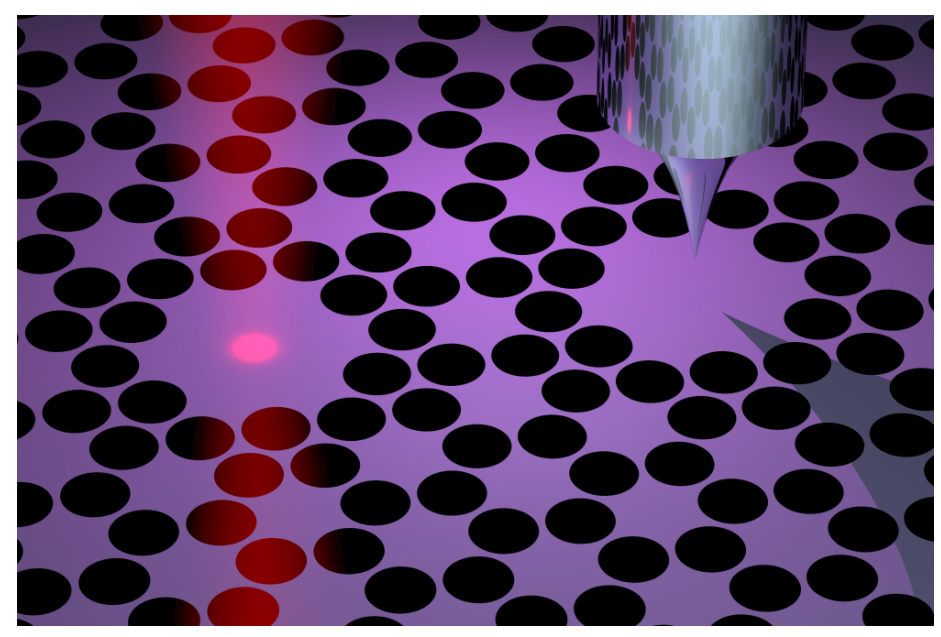

Physicists have come up with several ways to measure extremely small magnetic forces. One of the most sensitive techniques involves placing a tiny sample on a long, thin probe that acts as a "floppy" cantilever. By monitoring the vibrations of the cantilever, researchers can measure the force between the sample and a nearby magnet. Current cantilever systems can measure forces at roughly the 5-10 attonewton level (about the weight of a small strand of DNA). To measure the magnetic force of a single nucleus, a force sensor would need to reach the 0.1 attonewton level. Techniques that use carbon nanotubes have this force sensitivity, but the challenge is mounting the sample on the nanometer-wide tubes. "That's often the tension that we're working with," says Alexander Eichler from the Swiss Federal Institute of Technology in Zurich (ETHZ). "We want good force sensitivity, but at the same time, we want a design-friendly sensor that allows loading and manipulating of samples."

Eichler and his colleagues have developed a new force microscope that may overcome the difficulties of previous devices. Their idea is to invert the geometry by placing the sample on a vibrating membrane and bringing a probe next to it. In their design, the 40-nanometer-thick membrane is made of silicon nitride and is perforated with an array of holes that help to keep the vibrations steady, or "coherent." At the center of this array are two 100-micrometer-wide pads. The left pad is the target for laser light that both drives and monitors the

Brane scan. A new approach to force microscopy uses a thin membrane (purple), whose vibrations are controlled and monitored with laser light (left). A scanning probe moves over a separate section of the membrane (right), where samples are placed.

Credit: D. Hälg et al. [1] membrane's vibrations, while the right pad serves as the sample holder. The two pads vibrate in sync, so the team can measure the vibrations without exposing the sample to the laser light.

To demonstrate force microscopy, the team performed imaging scans of two types of samples: gold nanoparticles and virus 


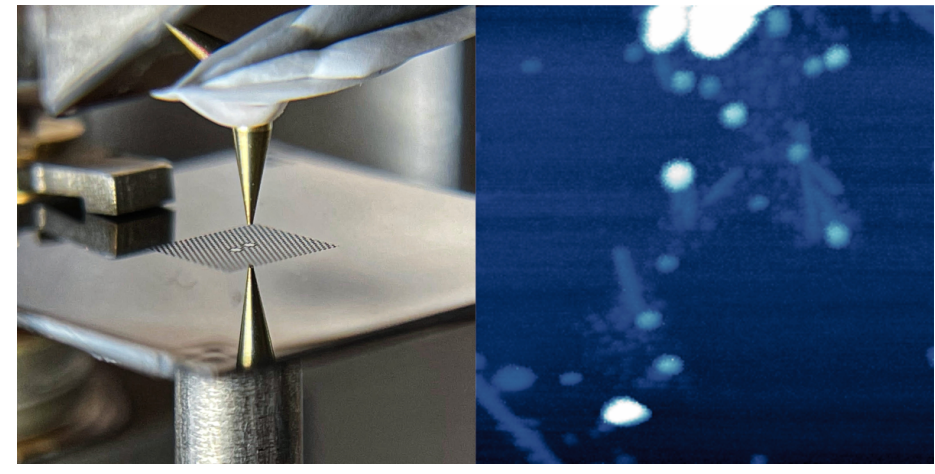

Feel the force. The experimental setup showing the membrane and probe holder (left) and a scanning microscope image of a mixture of gold nanospheres and virus particles (right).

Credit: D. Hälg and S. Misra/ETHZ

specimens. For each pixel in an image, the researchers placed the probe-an unmagnetized and ultrasharp "tip"-above the vibrating membrane and slowly lowered it down. When the probe got close to the sample, electrical interactions between the two materials caused the vibrations to change. By measuring the tip height where the vibrations changed and then repeating the measurement at each location, the team was able to map out the shapes of the samples.

However, imaging surfaces based on these electrical forces was just a demonstration. The team's ultimate goal is to produce a 3D image of a sample's interior by detecting the tiny oscillations in magnetic force from spins driven to flip by an external time-varying magnetic field. Such nano-MRI capability will require several changes to the system. The first is to improve the force sensitivity, which is currently around 280 attonewtons.
To potentially detect forces 1000 times smaller, the team is optimizing the membrane geometry and lowering the temperature below $1 \mathrm{~K}$. They are also planning to place the membrane in an optical cavity to improve the detection of vibrational changes.

Although it may be years away, the team imagines an instrument that could respond to an outbreak of a new viral disease by providing a 3D image of the virus in a matter of a few weeks. Current virus imaging methods take months and require complex sample preparation. Proteins are also hard to image. "In our technique, you would load one virus or one protein onto the membrane, and you could image it," says ETHZ graduate student David Hälg.

"The work pushes the frontiers of ultrasensitive detection in a new and exciting direction," says nano-MRI researcher Raffi Budakian from the University of Waterloo in Canada. The membrane system can be tailored in many different ways, since "you're not locked into a particular geometry or a particular frequency range," he says. Force sensing expert Tjerk Oosterkamp from Leiden University in the Netherlands says the large size of the membrane-compared to previous force sensors-could potentially allow relatively big samples, such as thinly cut tissues, to be imaged.

Michael Schirber is a Corresponding Editor for Physics based in Lyon, France.

\section{REFERENCES}

1. D. Hälg et al., "Membrane-based scanning force microscopy," Phys. Rev. Applied 15, L021001 (2021). 